\title{
Evolution of oxygen-metal electron transfer and metal electronic states during Mn-oxide catalyzed water oxidation revealed with in situ soft X-ray spectroscopy
}

\author{
Marc F. Tesch, ${ }^{[a, b]}$ Shannon A. Bonke, ${ }^{[c, d]}$ Travis E. Jones, ${ }^{*}[\mathrm{e}]$ Maryam N. Shaker,,${ }^{[a, f]}$ Jie Xiao, ${ }^{[a]}$ \\ Katarzyna Skorupska, ${ }^{[b, e]}$ Rik Mom, ${ }^{[e]}$ Jens Melder, ${ }^{[g]}$ Philipp Kurz, ${ }^{[g]}$ Axel Knop-Gericke, ${ }^{[e]}$ \\ Robert Schlögl, ${ }^{[b, e]}$ Rosalie K. Hocking, ${ }^{[h]}$ and Alexandr N. Simonov*[c]
}

Dedicated to Prof. Leone Spiccia - an outstanding scientist and personality who inspired this work.

\begin{abstract}
Manganese oxide $\left(\mathrm{MnO}_{\mathrm{x}}\right)$ electrocatalysts are examined herein by in situ soft X-ray absorption spectroscopy (XAS) and resonant inelastic X-ray scattering (RIXS) during the oxidation of water buffered by borate $(\mathrm{pH} 9.2)$ at potentials from 0.75 to $2.25 \mathrm{~V}$ vs. the reversible hydrogen electrode. Correlation of L-edge XAS data with previous mechanistic studies indicates $\mathrm{Mn}^{\mathrm{IV}}$ is the highest oxidation state involved in the catalytic mechanism. $\mathrm{MnO}_{\mathrm{x}}$ is transformed into birnessite at $1.45 \mathrm{~V}$ and does not undergo further structural phase changes. At potentials beyond this transformation, RIXS spectra show progressive enhancement of charge transfer transitions from oxygen to manganese. Theoretical analysis of these data indicates increased hybridization of the $\mathrm{Mn}-\mathrm{O}$ orbitals and withdrawal of electron density from the O ligand shell. In situ XAS experiments at the $\mathrm{O} \mathrm{K}$-edge provide complementary evidence for such a transition. This step is crucial for the formation of $\mathrm{O}_{2}$ from water.
\end{abstract}

[a] Dr. M. F. Tesch, M. N. Shaker, Dr. J. Xiao Institut Methoden der Materialentwicklung Helmholtz Zentrum Berlin für Materialien und Energie Albert-Einstein-Straße 15, 12489 Berlin, Germany

[b] Dr. M. F. Tesch, Dr. K. Skorupska, Prof. Dr. R. Schlögl Abteilung Heterogene Reaktionen

Max-Planck-Institut für Chemische Energiekonversion Stiftstraße 34-36, 45470, Mülheim an der Ruhr, Germany

[c] Dr. S. A. Bonke, Dr. A. N. Simonov

School of Chemistry and the ARC Centre of Excellence for Electromaterials Science

Monash University

Victoria 3800, Australia

E-mail: alexandr.simonov@monash.edu

[d] Dr. S. A. Bonke

Institut Nanospektroskopie

Helmholtz-Zentrum Berlin für Materialien und Energie

Kekuléstraße 5, 12489 Berlin, Germany

[e] Dr. T. E. Jones, Dr. K. Skorupska, Dr. Rik Mom,

Dr. A. Knop-Gericke, Prof. Dr. R. Schlögl

Abteilung Anorganische Chemie

Fritz-Haber-Institut der Max-Planck-Gesellschaft

Faradayweg 4-6, 14195 Berlin, Germany

E-mail: triones@fhi-berlin.mpg.de

[f] Freie Universität Berlin

Fachbereich Physik

Arnimallee 14, 14159 Berlin, Germany

[g] J. Melder, Prof. Dr. P. Kurz

Institut für Anorganische und Analytische Chemie and Freiburger Materialforschungszentrum, Albert-Ludwigs-Universität Freiburg

Albertstraße 21, 79104 Freiburg, Germany.

[h] Dr. R. K. Hocking

Department of Chemistry and Biotechnology,

Swinburne University of Technology,

John Street, Hawthorn, Victoria 3122, Australia
Electroreductive synthesis of fuels powered by renewable energy sources is globally recognized as a critical future technology needed to satisfy persistently surging energy demands. Such a synthesis requires an electron source of suitably large scale. An ideal option is water, the oxidation of which produces the electrons and protons that are necessary to synthesize fuels, as well as the useful by-product of $\mathrm{O}_{2}$. In order to reach significant current densities at moderate overpotentials, the electrochemical water oxidation reaction requires the use of catalysts. Most of these are based on transition metal oxides of which manganese oxides/oxyhydroxides $\left(\mathrm{MnO}_{\mathrm{x}}\right)$ are prominent examples. Although typically less active than catalysts based on the non-noble-metals nickel, iron or cobalt, ${ }^{[1]}$ manganese systems have advantages of abundance and lower toxicity. These considerations and inspiration from the biological catalyst for water-oxidation, the $\mathrm{CaMn}_{4} \mathrm{O}_{x}$ cluster of the enzyme Photosystem II, have spurred great interest in $\mathrm{MnO}_{x}$-catalyzed electrooxidation of $\mathrm{H}_{2} \mathrm{O}$ for renewable fuel synthesis. ${ }^{[2]}$

Remarkable progress in understanding the mechanistic aspects of water oxidation electrocatalysis has been achieved via three mutually complementary strategies: (i) density functional theory calculations to identify plausible reaction pathways, ${ }^{[3]}$ (ii) mechanistic kinetic studies for deciphering the mechanism, ${ }^{[4]}$ and (iii) spectroscopic analysis of catalytic materials to reveal transformations relevant to catalysis. ${ }^{[5]}$ For the latter, X-ray based techniques allowing comprehensive characterization of the structure and electronic properties of transition metal materials are of fundamental importance. A substantial body of valuable ex situ hard and soft $\mathrm{X}$-ray spectroscopic studies on $\mathrm{MnO}_{\mathrm{x}}$-catalyzed water oxidation exists (see ${ }^{[6]}$ and refs therein). However, probing the states of metal oxides involved in different stages of the catalytic cycle requires in situ spectroscopic measurements under electrocatalytic conditions, which are limited in number for $\mathrm{Mn}$ based catalysts. ${ }^{[5 a-d]}$

Hitherto, key in situ X-ray absorption spectroscopy (XAS) studies on $\mathrm{MnO}_{\mathrm{x}}$ were undertaken at the $\mathrm{Mn} \mathrm{K}$-edge using hard $\mathrm{X}$-rays, which is a powerful approach to decipher the structure and derive the average oxidation state of $\mathrm{Mn}$. At the same time, significantly deeper insights into the electronic structure of the metal-ligand (Mn-O) sites and electronic transitions can be obtained via soft XAS and resonant inelastic X-ray scattering (RIXS) at the $\mathrm{Mn} \mathrm{L}_{2,3^{-}}$ edges. Thus, a comprehensive in situ soft XAS and RIXS study of $\mathrm{MnO}_{\mathrm{x}}$ water oxidation catalysts is described herein. The spectra were collected with a wide range of potentials applied to the catalyst-modified electrodes, allowing changes in the state of 
$\mathrm{MnO}_{\mathrm{x}}$ to be correlated with the kinetics of the electrocatalytic reaction.

An equally important feature of the present work is the use of a fully parameterized model of $\mathrm{MnO}_{x}$-catalyzed $\mathrm{H}_{2} \mathrm{O}$ oxidation ${ }^{[4]}$ to define the potential needed to stabilize the catalyst in its putative active state (see Supporting Information (SI), Figure S1). Potentials examined in all previous studies were less positive than the critical value of $c a 2 \mathrm{~V} v s$. the reversible hydrogen electrode (RHE; all potentials are given vs. this reference hereinafter). Measurements at $>2 \mathrm{~V}$ are needed to exclude or reveal the involvement of higher oxidation states of $\mathrm{Mn}$ in the catalytic mechanism of water electrooxidation. ${ }^{[4]}$

To accommodate the requirements for a high-quality in situ L-edge XAS/RIXS electrochemical experiment, measurements were undertaken at the LiXEdrom end station of the BESSY II synchrotron facility using a custom-made flow cell (Scheme 1). Films of $\mathrm{MnO}_{x}$ were deposited by oxidative electrodeposition at an applied potential of $1.95 \mathrm{~V}$ on Au-coated $\mathrm{Si}_{3} \mathrm{~N}_{4}$ membranes from aqueous $\mathrm{Mn}^{2+}$ solutions buffered with borate $(0.1 \mathrm{M}, \mathrm{pH} 9.2)$. After deposition, a Mn-free borate buffer was used as a background electrolyte to obtain cyclic voltammograms and maintain the electrode at a constant potential during spectroscopic analysis. Further experimental details are provided in the SI.

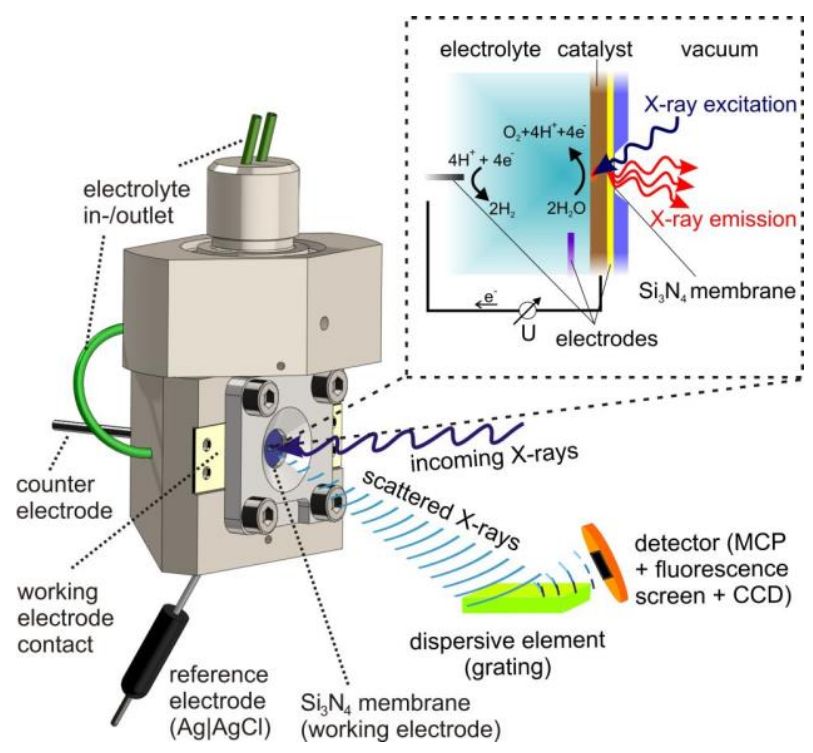

Scheme 1. Schematic representation of the flow cell used for the in situ XAS and RIXS experiments. Incoming X-rays penetrate the $100 \mathrm{~nm} \mathrm{Si}{ }_{3} \mathrm{~N}_{4}$ membrane and $20 \mathrm{~nm} \mathrm{Au}$ layer, interact with the $\mathrm{MnO}_{x}$ catalyst and generate the emitted/scattered photons that are measured in an energy resolved fashion. $\mathrm{MnO}_{\mathrm{x}}$ is in contact with an electrolyte solution. The potential applied to $\mathrm{MnO}_{\mathrm{x}} / \mathrm{Au}$ is controlled in a conventional three-electrode electrochemical configuration.

Figure 1 features representative cyclic voltammograms for the electrochemically deposited $\mathrm{MnO}_{\mathrm{x}}$ in comparison to the unmodified $\mathrm{Au}-\mathrm{Si}_{3} \mathrm{~N}_{4}$ support, with both showing an expected combination of redox processes. On its own, the support exhibits features typical of gold electrodes, viz. surface oxide formation commencing at ca $1.3 \mathrm{~V}$ and subsequent reduction as a welldefined peak at ca $1.0 \mathrm{~V}$. Functionalization of the electrode with
$\mathrm{MnO}_{x}$ suppresses these signals and produces a voltammetric curve dominated by processes associated with the water oxidation catalyst: (i) a combination of at least two redox transformations of $\mathrm{Mn}$ within the potential range $0.8-1.4 \mathrm{~V} \mathrm{~V}^{[4]}$ and (ii) the electrocatalytic oxidation of $\mathrm{H}_{2} \mathrm{O}$ at potentials $>1.6 \mathrm{~V}$. A mismatch between the anodic and cathodic counterparts of the voltammogram within this catalytic region is interpreted in terms of mass-transport limitations for the $\left[\mathrm{H}_{2} \mathrm{BO}_{3}\right]^{-/}\left[\mathrm{B}(\mathrm{OH})_{4}\right]^{-}$conjugate base as defined by the experimental conditions employed, viz. no convection, comparatively low borate concentration, and a small solution volume.

In situ XAS/RIXS analysis was undertaken at nine potentials with special attention paid to the catalytic region (marked by dashed lines in Figure 1). The ground-state of the catalyst was probed with no potential applied after cyclic voltammetric analysis of freshly deposited $\mathrm{MnO}_{\mathrm{x}}$. XAS data were obtained in partial fluorescence yield (PFY) mode (Figure 2). We have also undertaken ex situ Mn L-edge XAS analysis in total electron yield (TEY) mode of as-deposited $\mathrm{MnO}_{\mathrm{x}}$ and after conditioning at different potentials to confirm previous findings (Figure S2). ${ }^{[5]}$ Both in situ PFY and ex situ TEY methods reveal typical multiplet structures at the $\mathrm{Mn}_{3}$ (ca 639-647 eV) and $M n \mathrm{~L}_{2}$ (ca 650-657 $\mathrm{eV}$ ) edges, stemming from transitions of the $2 p$ core levels to the $3 d$ conduction band (valence states) and subsequent relaxation via electron/photon emission.

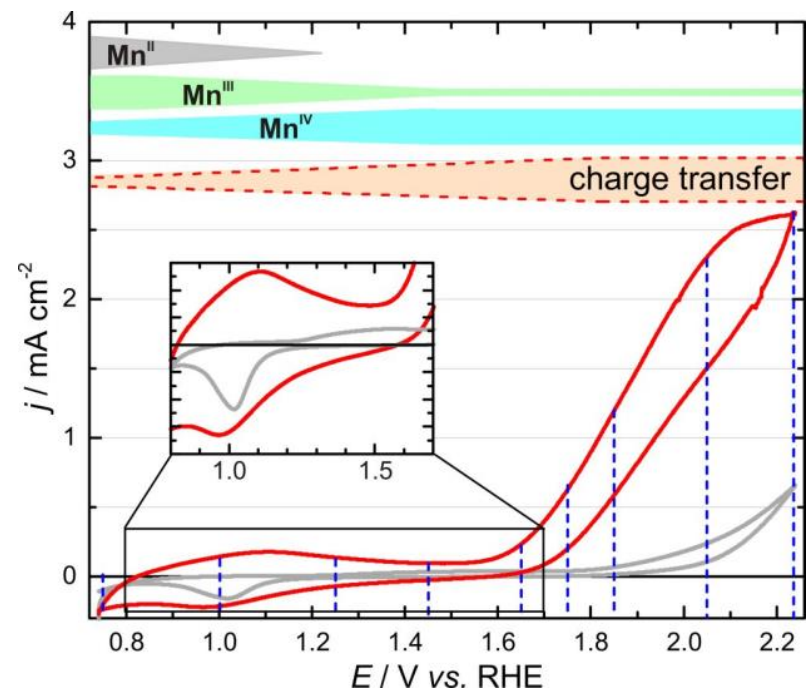

Figure 1. Cyclic voltammograms (scan rate $0.02 \mathrm{~V} \mathrm{~s}^{-1}$ ) for electrodeposited $\mathrm{MnO}_{\mathrm{x}}(\mathrm{red})$ and unmodified $\mathrm{Au} / \mathrm{Si}_{3} \mathrm{~N}_{4}$ (gray) in $0.1 \mathrm{M}$ borate buffer $(\mathrm{pH} 9.2$ ) at $23 \pm 2{ }^{\circ} \mathrm{C}$. The inset shows an enhanced plot of the outlined region. Bars with oxidation states illustrate the evolution of Mn oxidation states derived from spectroscopic data herein. Dashed lines show potentials used for in situ spectroscopic analysis. Currents are normalized to the geometric surface area of the electrode.

Application of increasingly positive potentials from 0.75 to $2.25 \mathrm{~V}$ induces significant changes to the shape of the XA spectra (Figure 2 and Figure S2). For the $L_{3}$ edge, peaks at 641.6 (mainly associated with $\mathrm{Mn}^{\mathrm{III}}$ ) and especially $640.2 \mathrm{eV}\left(\mathrm{Mn}^{\mathrm{II}}\right)$ are increasingly suppressed, while the features at 640.8 and 643.7 $\mathrm{eV}$ (fingerprints of $\mathrm{Mn}^{\mathrm{IV}}$ ) notably enhance in intensity. At the $\mathrm{L}_{2}$ 
edge, the whole multiplet shifts towards higher energies. The observed spectral changes are consistent with an increase of the formal $\mathrm{Mn}$ oxidation state at more positive potentials, ${ }^{[7]}$ and can be further correlated with structural transformations within the $\mathrm{MnO}_{\mathrm{x}}$ catalyst films. The TEY data obtained for $\mathrm{MnO}_{\mathrm{x}}$ are perfectly reproduced as a linear combination of the spectra for powder reference materials (Figure S4). However, Mn L-edge XAS is most sensitive to the differences in electronic states of the metal and might not provide unambiguous distinction between different phases in multicomponent materials. Hence, these data are mainly used herein to establish the effects of potential on the manganese oxidation state. On the basis of this type of analysis, the as-deposited $\mathrm{MnO}_{x}$ can be formally considered as a mixture of $\mathrm{Mn}_{3} \mathrm{O}_{4}, \mathrm{Mn}_{2} \mathrm{O}_{3}$ and birnessite in near equal amounts, which is oxidized to produce a material dominated by $\mathrm{Mn}_{2} \mathrm{O}_{3}$ and birnessite upon conditioning at $1.25 \mathrm{~V}$. At $1.45 \mathrm{~V}$ and $1.75 \mathrm{~V}$, the spectra of $\mathrm{MnO}_{x}$ strongly resemble those obtained for a birnessite standard, possibly with a minor contribution from $\mathrm{MnO}_{2}$ (Figure S4), which accords with previous reports ${ }^{[2 a, 5 d, 5 e]}$ and theoretical analysis (vide infra). Crucially, the TEY measurements were undertaken ex situ making it probable that the reactive species were reduced prior analysis. Importantly, the changes summarized above are reversible as confirmed by measurements undertaken with potentials switched in a random sequence.

When analysis is undertaken in situ, the PFY spectra contain features identical to those obtained in TEY mode (except for the well-known enhancement of the $\mathrm{L}_{2}$-edge in $\mathrm{FY}^{[8]}$ ) suggesting no $X$-ray beam damage to the samples, and the phase formed at positive potentials is therefore also identified as birnessite. The potential dependent evolution of the in situ spectra resembles the behavior observed in the ex situ experiments (cf. Figure 2 and Figure S2), but suggests deeper conversion to birnessite at less positive potentials. PFY is bulk sensitive (ca 10-100 nm), and strong signal changes upon oxidation of $\mathrm{MnO}_{x}$ attest to the complete transformation of the catalyst into birnessite. Upon oxidation at $1.75 \mathrm{~V}$, the morphology of electrodeposited $\mathrm{MnO}_{x}$ also changed as detected by scanning electron microscopy (Figure S5); the initially present mixed flakes and grains were transformed into homogeneous flakes that more uniformly cover the support surface.

Thus, the data in Figure 2 infer that no significant changes to the oxidation state of $\mathrm{MnO}_{\mathrm{x}}$ occur at potentials more positive than $1.45 \mathrm{~V}$, as alterations in the shape and position of both the $\mathrm{L}_{3}$ and $L_{2}$ multiplets are negligible. This observation is concordant with Mn K-edge XAS. ${ }^{\left[{ }^{[d]}\right]}$ However, the catalyst became highly prone to $X$-ray induced damage whenever strongly oxidative potentials were applied, viz. $>1.75 \mathrm{~V}$ (this was addressed by reducing the photon flux to enable collection of reliable spectroscopic data as explained in the SI). Moreover, this potential- plus X-ray-induced degradation produces $\mathrm{Mn}^{\|}$resonances in the XA spectra (Figure S6). This behavior is indicative of the generation of a highly reactive state of $\mathrm{MnO}_{x}$ at $>1.75 \mathrm{~V}$, which is readily reduced by $\mathrm{X}$-rays into soluble species. It is important to emphasize that no beam damage was found during in situ measurements at $\leq 1.75 \mathrm{~V}$ at 10x higher X-ray intensity. Nevertheless, even L-edge XAS did not provide the necessary level of sensitivity to probe this labile $\mathrm{MnO}_{\mathrm{x}}$ state, which incited us to undertake an in situ RIXS study.

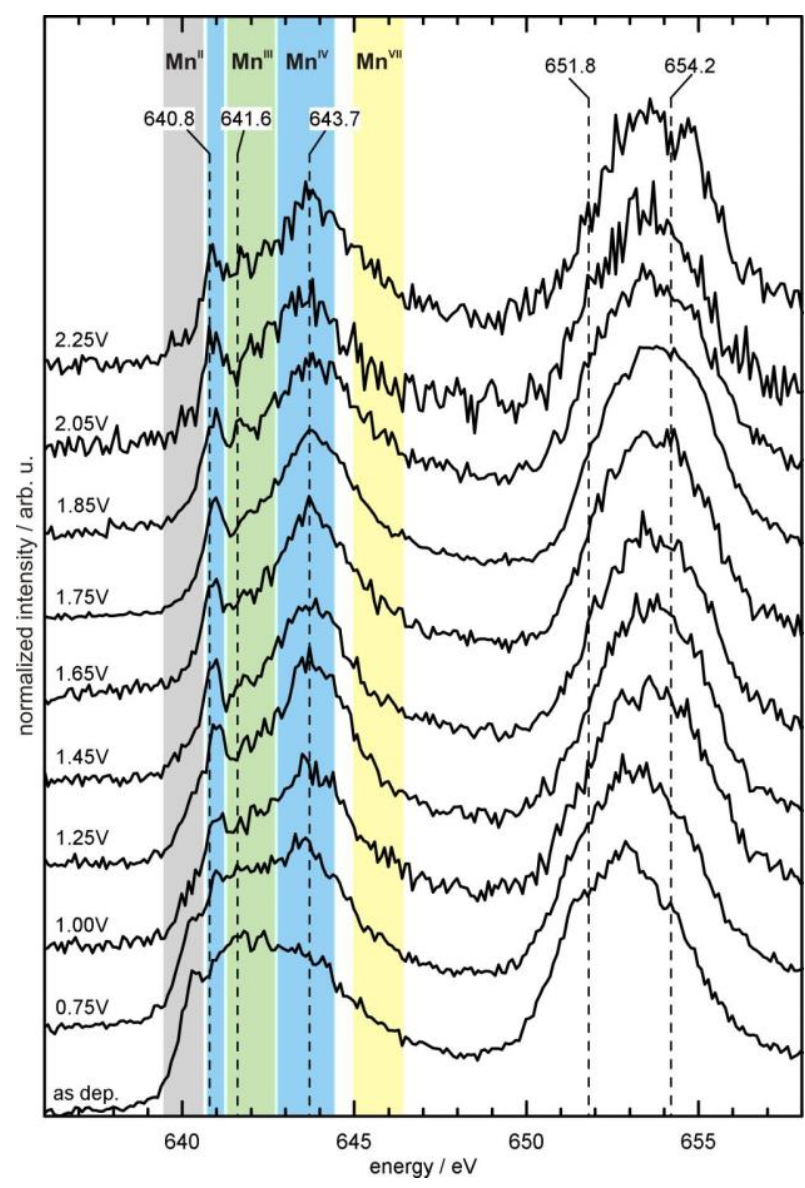

Figure 2. In situ absorption spectra at the $\mathrm{Mn}_{2,3}$-edges measured in PFY mode, viz. $2 p \rightarrow 3 d$ transitions and subsequent photon emission by recombination, for $\mathrm{MnO}_{x}$ in borate buffer $(0.1 \mathrm{M}, \mathrm{pH}$ 9.2) obtained after deposition with no potential applied (as dep.) and at the specified potentials reported vs. RHE. Dashed lines show energies used to collect RIXS spectra. Background shading shows the spectral regions prominently corresponding to particular Mn oxidation states. ${ }^{[7]}$ Corresponding current-time transients are exemplified in Figure S3. Data were obtained at $23 \pm 2{ }^{\circ} \mathrm{C}$ and normalized to a maximum intensity of 1 .

Figure 3 shows RIXS data obtained at different potentials (dashed lines in Figure 1) as energy loss spectra of scattered photons when irradiating the electrode with X-rays of a chosen energy (dashed lines in Figure 2). The energy uptake of the system due to inter- and intra-atomic electronic transitions can thus be probed. All spectra exhibit a peak from elastically scattered photons that did not transfer energy to the system, and hence appear at zero energy loss. Other spectral features result from an interplay of $d d$ excitations and charge transfer transitions occurring at essentially constant energy loss independent of the excitation energy, as well as fluorescence features with positions that scale linearly with the excitation energy (for further details on the principles of RIXS see relevant reviews, e.g. $\left.{ }^{[9]}\right)$. The need to employ low-intensity X-ray flux so as to avoid the photoreduction of $\mathrm{Mn}$ eventuated in a comparatively low RIXS signal to noise ratio. To compare the spectral features, a noise background signal was first subtracted from each spectrum and all spectra were subsequently normalized to an integrated intensity of 1 (see Data Interpretation section in the $\mathrm{SI}$ ). 
The energies used to record the RIXS data were chosen to cover the regions where the PFY spectra exhibited the most pronounced potential-induced changes (Figure 2), which reflect significant alterations in the electronic structure of the unoccupied states. Within the potential range examined, the RIXS spectra recorded at the $\mathrm{Mn}_{3}$-edge $(640.8,641.6$ and $643.7 \mathrm{eV})$ and the $\mathrm{L}_{2}$-edge (651.8 and $654.2 \mathrm{eV}$ ) exhibit prominent and clearly distinguishable features at 3-4 eV and 6-8 eV energy loss (Figure $3)$. The RIXS spectra at the $L_{2}$-edge additionally develop new signals with energy losses above $10 \mathrm{eV}$ that concertedly shift in position when changing the excitation energy (Figure 3). Such behavior is typical of fluorescence peaks. ${ }^{[9]}$

The features appearing at constant energy loss, independent of the excitation energy, are typical of $d d$ excitations (ca 1-5 eV), and charge transfer transitions (ca 5-10 eV). Interestingly, their energetic positions in the photon energy loss spectra are only marginally influenced by the applied potential (Figure 3 ). This observation suggests that the energies needed for a $d d$ transition or a charge transfer are similar for all Mn oxidation states involved. Invariability in the position of the $d d$ transition peak with the lowest energy loss at ca $3 \mathrm{eV}$ indicates that the applied potentials do not induce a significant change in the band gap.

At the same time and most importantly, application of potential to the $\mathrm{MnO}_{\mathrm{x}}$ films produces notable changes in the intensity of the charge transfer peaks (Figures 3 and S9). The strongest potentialinduced effects are found in the RIXS spectra collected at low energies of the $\mathrm{Mn}_{3}$-edge ( 640.8 and $641.6 \mathrm{eV}$ ). Specifically, the intensity of charge transfer features, which is low relative to the $d d$ transition signals for the as-deposited state and at $0.75 \mathrm{~V}$, progressively enhances as more positive potentials are applied. Deeper insights into this phenomenon were obtained via experiment-simulation comparisons.

Simulations of the $\mathrm{XA}$ spectrum of $\mathrm{Mn}^{\mathrm{IV}}$ in a birnessite geometry were able to reproduce all experimentally observed features (Figure S10), thus verifying the appropriateness of the employed level of theory (full details of our approach based on a configuration interaction model are provided in the SI). Upon absorption of $640.8 \mathrm{eV}$ photons, simulations support the excitation of electrons into $\mathrm{Mn}^{\mathrm{IV}} \sigma\left(\mathrm{e}_{\mathrm{g}}\right)$ orbitals, which are empty for the $3 d^{\beta}$ electronic configuration and octahedral geometry (Figure S11). Notably, simulations that do not account for orbital hybridization may produce XA spectra similar to those experimentally observed (Figure S12), but cannot reproduce the RIXS peaks at ca 5-10 eV (Figure S13). However, the simulated RIXS spectra show charge transfer features in the 5-10 eV range when accounting for orbital hybridization in the model (Figure S14), concordant with the experimental data and verifying the origin of these peaks. Specifically, theory indicates a ligand to metal charge transfer from an $\mathrm{O} 2 p$ into a $\mathrm{Mn} 3 d$ orbital. Considering crystal field theory and the octahedral geometry, electron transfer is expected to occur into the $M n \sigma\left(e_{g}\right)$ orbitals due to the two-fold greater orbital overlap compared to the $\pi\left(t_{2 g}\right)$ states. This is also supported in simulations by theoretically allowing or disallowing electron transfer into either the $\mathrm{Mn} \sigma$ or $\pi$ orbitals (Figure S15).

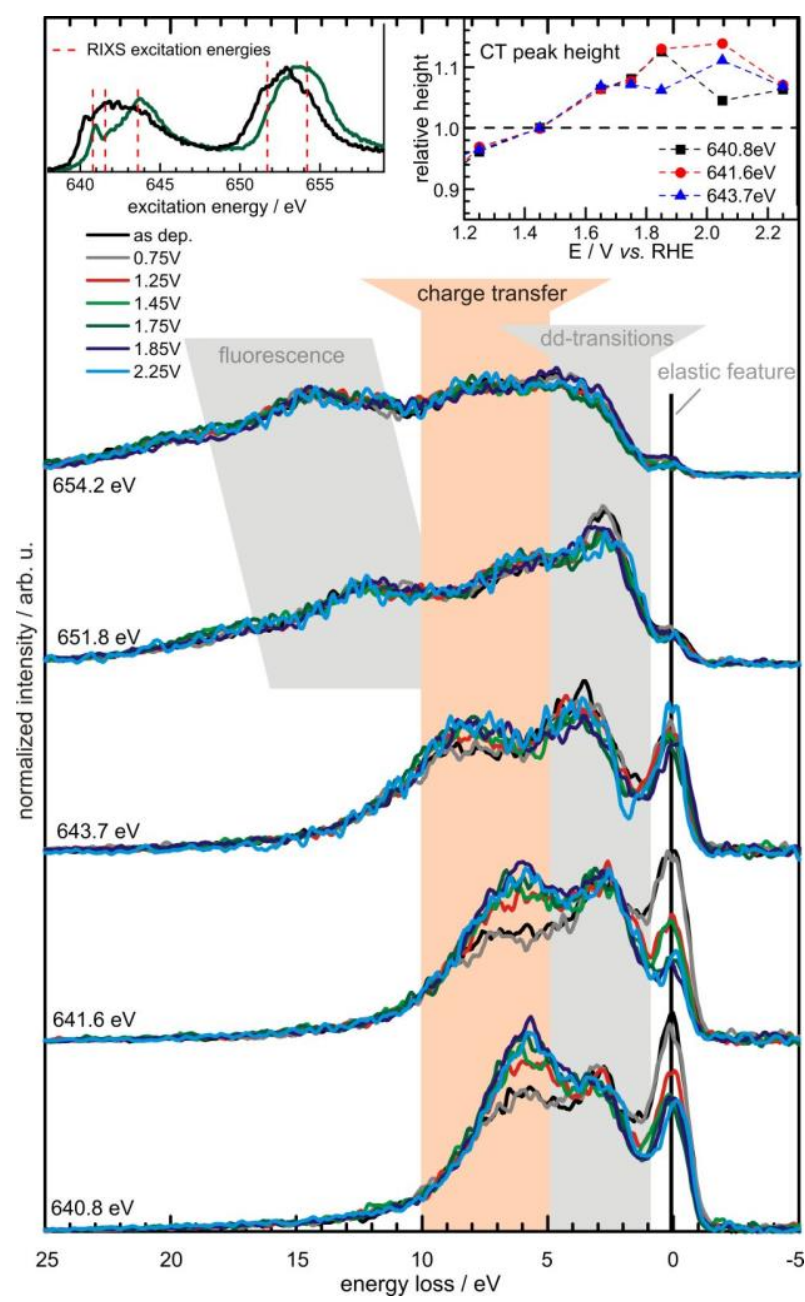

Figure 3. In situ energy loss spectra of scattered photons (RIXS) obtained a $23 \pm 2{ }^{\circ} \mathrm{C}$ for $\mathrm{MnO}_{x}$ in $0.1 \mathrm{M}$ borate buffer $(\mathrm{pH}$ 9.2) with no potential applied (black) and at 0.75 (gray), 1.25 (red), 1.45 (light green), 1.75 (dark green), 1.85 (dark blue), and $2.25 \mathrm{~V}$ vs. RHE (turquoise). Data were background corrected and normalized to an integrated intensity of 1 . Energies of the incident X-rays were 640.8, 641.6, 643.7, 651.8 and 654.2 eV (relevant parts of the PFY spectra are shown in the left inset for as-deposited (black) and polarized at $1.75 \mathrm{~V} \mathrm{MnO}$ (dark green). Features of different origins discussed in the text are highlighted with background colors. The relative heights of the charge transfer (CT) features compared to the spectra taken at $1.45 \mathrm{~V}$ are shown in the right inset for the $640.8,641.6$, and $643.7 \mathrm{eV}$ excitation energies. Data at all potentials examined (Figure S7), and a 2D RIXS map for $\mathrm{MnO}_{x}$ as-deposited and at $1.65 \mathrm{~V}$ (Figure $\mathrm{S} 8)$, are shown in the SI.

The efficiency of charge transfer in first-row transition metal complexes is strongly linked to orbital hybridization, ${ }^{[10]}$ which was probed theoretically herein by varying the degree of hybridization of $\mathrm{Mn} 3 d$ and $\mathrm{O} 2 p$ orbitals. Within the framework of the employed model, this variation was achieved by altering the energy difference between the two orbitals $(\Delta)$, and the Mn-O bond length via the extent of orbital wavefunction overlap (the O-Mn hopping integral, $V_{\text {eg }}$ ). A decrease in $\Delta$ induces greater orbital hybridization, as does an increase in $V_{\text {eg. }}$. Importantly, changes in these parameters had minimal effect on the simulated birnessite XA spectra (see Figure S10 and discussions in SI), consistent with the potential independent behavior of the experimental XAS data at $>1.45 \mathrm{~V}$ (Figure 2). In contrast, the measured RIXS spectra 
display clear intensity changes over this potential range (Figure 3 ), which can be mimicked by varying the degree of orbital hybridization in the simulations. Indeed, decreasing $\Delta$ increases the intensity of the charge transfer peaks, although this also shifts the peak position to lower energy loss, in disagreement with experimental observations (Figure S16). This is expected, as $\Delta$ defines the energy of the ligand to metal charge transfer. Meanwhile, higher $V_{\text {eg }}$ also induces greater hybridization, but shifts the peak position in the opposite direction (Figure S17). Thus, the experimental observations can be simulated with a combination of these two parameters that both reflect enhanced hybridization, viz. a smaller energy difference between the $\mathrm{Mn} 3 d$ and $\mathrm{O} 2 p$ orbitals along with a Mn-O bond length contraction (Figure S18). The relative intensity of the charge transfer feature increased by $8-10 \%$ upon changing the applied potential from 1.45 to $\geq 1.75 \mathrm{~V}$ in the experimental spectra (Figure 3 ). This can be simulated using a heuristic approach by decreasing $\Delta$ on the order of an electron volt, combined with an increase in $V_{\text {eg }}$ of a few hundred meV. These changes are indicative of a withdrawal of electron density from the $\mathrm{O}$ ligand shell (a comprehensive discussion is included in $\mathrm{SI}$ ). This change in $\Delta$ can be ascribed to an increase of $\mathrm{Mn} 3 d$ and $\mathrm{O} 2 p$ orbital hybridization leading to significant ligand hole character, which would correspond to a formal oxidation state of -1 of the oxygen ligand $\left(d^{4} \underline{L}^{1}\right)$, rather than $\mathrm{O}^{2-}\left(\mathrm{d}^{3} \underline{\underline{L}}^{0}\right)$.

The RIXS results reported herein correlate with prominent mechanistic proposals for water oxidation by the $\mathrm{CaMn}_{4} \mathrm{O}_{\mathrm{x}}$ cluster in photosystem $1 \mathrm{I},{ }^{[11]}$ as well as in situ X-ray photoelectron spectroscopic and $\mathrm{O}$ K-edge XAS studies on $\mathrm{IrO}_{2}$ electrocatalysts, ${ }^{[12]}$ with the generation of $\mathrm{O}^{-}$species having been suggested to precede the $\mathrm{O}-\mathrm{O}$ bond formation in both cases. Measurements at the $\mathrm{O} \mathrm{K}$-edge should also provide evidence for the $\mathrm{O} 2 p$ hole formation detected by RIXS. This was achieved herein with the use of a setup that allows in situ XAS measurements in total electron yield mode (detailed description in the SI and schematic in Figure S19). ${ }^{[12]}$ The key feature of this setup is the separation of the working electrode from the solution with a Nafion membrane to provide a very thin layer of water through which photoelectrons can pass. Consequently, the in situ cell arrangements were very different for the RIXS and O K-edge measurements.

The use of the different cell layout (as shown in Figure S19) allowed chemically synthesized birnessite (SEM and XRD data in Figure S20) to be probed while enabling a measurable water electrooxidation rate (Figure S21a). Under these conditions, in situ XAS at the Mn L-edges confirmed that Mn oxidation states above IV are not formed (Figure S21b), which is consistent with results from the other in situ cell configuration (Figure 2). At the same time, the $\mathrm{OK}$-edge spectra undergo continuous changes as more positive potentials are applied (Figure 4). Specifically, the resonance at $529 \mathrm{eV}$ associated with transitions into locally unoccupied $\mathrm{O} 2 p$ states hybridized with the lowest energy Mn $3 d$ states (spin-majority $e_{\mathrm{g}}$ and $t_{2 \mathrm{~g}}$ ) $^{[12]}$ enhance in intensity. At the same time, the resonance at $532 \mathrm{eV}$ associated with oxidized graphene (Figure S21c) and $O 2 p$ states hybridized with the higher energy $\mathrm{Mn}$ states (spin-minority $\left.e_{g}\right)^{[13]}$ remains constant. Such behavior is indicative of a substantial increase in $\pi$ covalency by donation of an electron from $\mathrm{O}^{2-}$ to a $\mathrm{Mn}^{\mathrm{IV}}$ orbital (increased $\mathrm{O} 2 p$ hole character in $\mathrm{MnO}_{\mathrm{x}}$ ). It is also similar to the behavior of $\mathrm{IrO}_{x}$ during water oxidation catalysis. ${ }^{[11]}$ Crucially, this result is in full agreement with the aforesaid RIXS analysis. However, fundamental differences in the experimental conditions used for the two types of experiments do not allow direct quantitative correlations between them.

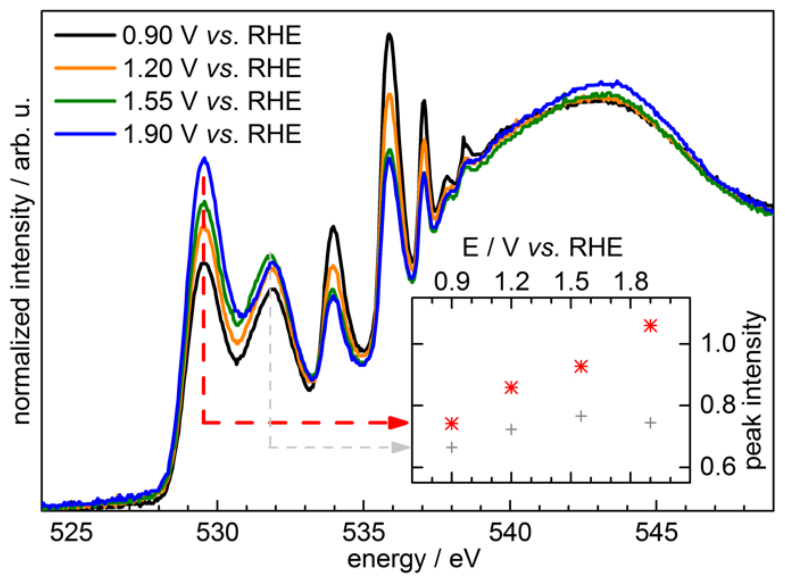

Figure 4. In situ $\mathrm{O}$ K-edge $\mathrm{XA}$ spectra obtained at $23 \pm 2{ }^{\circ} \mathrm{C}$ for birnessite in contact with a Nafion membrane impregnated with $0.1 \mathrm{M}$ phosphate buffer $(\mathrm{pH} 7.0)$ at 0.90 (gray), 1.20 (red), 1.55 (light green), and $1.90 \mathrm{~V}$ vs. RHE (dark blue). Inset shows the intensity at 529 and $532 \mathrm{eV}$ as a function of potential. Data were recorded in the cell shown in Figure S19.

Finally, the RIXS/XAS spectroscopic data from the Mn L-edges can be directly linked to the mechanistic electrochemical study that guided the in situ analysis herein. To reiterate, XAS indicates that the highest $\mathrm{Mn}$ oxidation state of IV is reached at $1.45 \mathrm{~V}$ (Figure 2), whereas the RIXS charge transfer peak intensity increases until the application of $1.75 \mathrm{~V}$ (Figure 3). These saturation values correlate well with the potentials of redox processes for $\mathrm{MnO}_{\mathrm{x}}$ electrocatalysts detected by Fourier transformed alternating current voltammetry. ${ }^{[4]}$ Therein, the last step for the oxidation of $\mathrm{MnO}_{\mathrm{x}}$ prior to the onset of water oxidation occurs at ca $1.5 \mathrm{~V}$ and is not directly involved in catalysis. Based on the PFY spectra, this transition can now be related to the complete transformation of the catalyst into birnessite, which is the catalytically active phase of $\mathrm{MnO}_{\mathrm{x}}$. At the same time, the ac voltammetric signal directly coupled to the oxidation of $\mathrm{H}_{2} \mathrm{O}$ is expected in the 1.70-1.75 $\mathrm{V}$ range (cf. water oxidation activity in Figure 1 and extrapolation of data in Figure $5 \mathrm{~b}$ from Ref. ${ }^{\left[{ }^{[4]}\right)}$, which is consistent with the stabilization of the RIXS data upon reaching these potentials (Figures 3 and S9). This indicates that $\mathrm{MnO}_{\mathrm{x}}$-catalyzed water oxidation requires facile charge transfer from $\mathrm{O}$ to $\mathrm{Mn}$, which is achieved at ca $1.75 \mathrm{~V}$ and does not intensify with the application of more positive potentials (up to $2.25 \mathrm{~V}$ ). Moreover, the aggregate of the XAS/RIXS data obtained herein and the outcomes of the preceding mechanistic study ${ }^{[4]}$ indicate that $\mathrm{Mn}^{\mathrm{IV}}$ is the highest oxidation state involved in water oxidation electrocatalysis.

In summary, the present study demonstrates how in situ soft XAS and RIXS guided by comprehensive electrochemical analysis and supported by theoretical simulations consolidate into a truly powerful modus operandi for probing electrocatalytic mechanisms. Herein, this strategy was employed to investigate 
water oxidation catalyzed by $\mathrm{MnO}_{x}$ under turnover conditions. It is demonstrated that: (i) birnessite is the catalytically active structural phase of $\mathrm{MnO}_{x}$ that does not undergo changes beyond ca $1.45 \mathrm{~V}$; (ii) there is no evidence supporting involvement of manganese species with oxidation states higher than IV to the catalytic mechanism; and (iii) the degree of hybridization of $\mathrm{Mn} 3 d$ and $\mathrm{O} 2 p$ orbitals increases with positive potentials up to $1.75 \mathrm{~V}$ and thereby $\mathrm{O}-\mathrm{Mn}$ charge transfer becomes increasingly facile. The latter potential correlates with the potential of the ratedetermining electron transfer step. ${ }^{[4]}$ Importantly, the extent of orbital hybridization detected by in situ RIXS and quantified via theoretical simulations suggests that the ligand-metal charge transfer is associated with the formation of an $\mathrm{O}^{-}$species. In situ $\mathrm{O}$ K-edge measurements verify the occurrence of this charge transfer. Thus, a crucial step in the interaction between an electrocatalyst and intermediates of water electrooxidation has been experimentally probed and correlated with the ratedetermining electron transfer process.

\section{Experimental Section}

Full experimental details are provided in the Supporting Information.

\section{Acknowledgements}

The authors are grateful to Dr. R. Golnak for assistance during the XAS/RIXS synchrotron beamtime, Mr. I. Rudolph for Aucoating of the $\mathrm{Si}_{3} \mathrm{~N}_{4}$ membranes, Mr. F. Siewert and Mrs. C. Klimm for microscopic characterization, Dr. C. Schwanke for assistance with the flow cell, Dr. K. Atak for scientific discussion related to theory and modeling, Prof. Dr. Anna Fischer for access to and assistance with the BMBF-funded HR-SEM at the ALU Freiburg (project EDELKAT, 03X5524), and Dr. A. Schnegg for support throughout the study. SAB and ANS acknowledge funding by the Australian Research Council via ACES (CE140100012); SAB acknowledges funding from the DFG through SPP 1601; MNS thanks the DAAD for the award of a doctoral scholarship (91527148); SAB and TEJ acknowledge the Alexander-von-Humboldt foundation for financial support; ANS is grateful to the Australian Synchrotron for funding through the International Synchrotron Access Program (ISP10692); MFT, KS, RM, JM, PK, AK-G and RS acknowledge financial support by the Federal Ministry of Education and Research (BMBF cluster project MANGAN, FKZ 03EK3545, 03SF0513 and 03SF0511A). We also thank Helmholtz-Zentrum Berlin and FHI for supporting the ISISS beamline at BESSY II.
Keywords: water electrooxidation $\cdot$ manganese oxide $\cdot$ RIXS • soft XAS • in situ spectroscopy

[1] R. Subbaraman, D. Tripkovic, K.-C. Chang, D. Strmcnik, A. P. Paulikas, P. Hirunsit, M. Chan, J. Greeley, V. Stamenkovic, N. M. Markovic, Nat Mater 2012, 11, 550-557.

[2] a) R. K. Hocking, R. Brimblecombe, L.-Y. Chang, A. Singh, M. H. Cheah, C. Glover, W. H. Casey, L. Spiccia, Nat Chem 2011, 3, 461 466; b) M. Wiechen, M. M. Najafpour, S. I. Allakhverdiev, L. Spiccia, Energy Environ Sci 2014, 7, 2203-2212.

[3] a) I. C. Man, H.-Y. Su, F. Calle-Vallejo, H. A. Hansen, J. I. Martínez, N. G. Inoglu, J. Kitchin, T. F. Jaramillo, J. K. Nørskov, J. Rossmeisl, ChemCatChem 2011, 3, 1159-1165; b) H.-Y. Su, Y. Gorlin, I. C. Man, F. Calle-Vallejo, J. K. Nørskov, T. F. Jaramillo, J. Rossmeisl, PCCP 2012, 14, 14010-14022.

[4] S. A. Bonke, A. M. Bond, L. Spiccia, A. N. Simonov, J Am Chem Soc 2016, 138, $16095-16104$.

[5] a) M. Yoshida, T. Yomogida, T. Mineo, K. Nitta, K. Kato, T. Masuda H. Nitani, H. Abe, S. Takakusagi, T. Uruga, K. Asakura, K. Uosaki, H. Kondoh, Chem Commun 2013, 49, 7848-7850; b) Y. Gorlin, B. Lassalle-Kaiser, J. D. Benck, S. Gul, S. M. Webb, V. K. Yachandra, J. Yano, T. F. Jaramillo, J Am Chem Soc 2013, 135, 8525-8534; c) M. Yoshida, T. Yomogida, T. Mineo, K. Nitta, K. Kato, T. Masuda, H. Nitani, H. Abe, S. Takakusagi, T. Uruga, K. Asakura, K. Uosaki, H. Kondoh, J Phys Chem C 2014, 118, 24302-24309; d) Zaharieva, D. Gonzalez-Flores, B. Asfari, C. Pasquini, M. R. Mohammadi, K. Klingan, I. Zizak, S. Loos, P. Chernev, H. Dau, Energy Environ Sci 2016, 9, 2433-2443; e) M. Khan, E. Suljoti, A Singh, S. A. Bonke, T. Brandenburg, K. Atak, R. Golnak, L. Spiccia E. F. Aziz, J Mater Chem A 2014, 2, 18199-18203.

[6] C. H. M. van Oversteeg, H. Q. Doan, F. M. F. de Groot, T. Cuk, Chem Soc Rev 2017, 46, 102-125.

[7] L. A. J. Garvie, A. J. Craven, Phys Chem Miner 1994, 21, 191-206.

[8] a) F.M.F. de Groot, M.A. Arrio, Ph. Sainctavit, Ch. Cartier, C.T. Chen, Solid State Commun 1994, 92, 991-995; b) R. Kurian, K. Kunnus, P. Wernet, S. M. Butorin, P. Glatzel, F. M. F. de Groot, J Phys: Condens Matter 2012, 24, 452201

[9] L. J. P. Ament, M. van Veenendaal, T. P. Devereaux, J. P. Hill, J. van den Brink, Rev Mod Phys 2011, 83, 705-767.

[10] M. N. Grisolia, J. Varignon, G. Sanchez-Santolino, A. Arora, S. Valencia, M. Varela, R. Abrudan, E. Weschke, E. Schierle, J. E. Rault, J. P. Rueff, A. Barthelemy, J. Santamaria, M. Bibes, Nat Phys 2016, 12, 484-492.

[11] a) P. E. M. Siegbahn, Acc Chem Res 2009, 42, 1871-1880; b) L. Rapatskiy, N. Cox, A. Savitsky, W. M. Ames, J. Sander, M. M. Nowaczyk, M. Rögner, A. Boussac, F. Neese, J. Messinger, W Lubitz, J Am Chem Soc 2012, 134, 16619-16634; c) N. Cox, M. Retegan, F. Neese, D. A. Pantazis, A. Boussac, W. Lubitz, Science 2014, 345, 804

[12] a) V. Pfeifer, T. E. Jones, J. J. Velasco Velez, C. Massue, M. T. Greiner, R. Arrigo, D. Teschner, F. Girgsdies, M. Scherzer, J. Allan, M. Hashagen, G. Weinberg, S. Piccinin, M. Havecker, A. KnopGericke, R Schlögl, Phys Chem Chem Phys 2016, 18, 2292-2296; b) V. Pfeifer, T. E. Jones, J. J. Velasco Velez, R. Arrigo, S. Piccinin, M. Havecker, A. Knop-Gericke, R. Schlögl, Chem Sci 2017, 8, 2143 2149.

[13] F. M. F. de Groot, M. Grioni, and J. C. Fuggle, J. Ghijsen and G. A Sawatzky, H. Petersen, Phys. Rev. B 1989, 40, 5715-5723. 
